\title{
ANÁLISIS DE FACTORES ASOCIADOS AL FENÓMENO DE LAS DROGAS EN ESTUDIANTES DE SEGUNDO Y CUARTO AÑO DE LA CARRERA DE LICENCIATURA EN ENFERMERÍA
}

\section{ANALYSIS OF FACTORS ASSOCIATED WITH THE DRUG PHENOMENON IN STUDENTS OF SECOND AND FOURTH YEAR OF "LICENCIATURA EN ENFERMERÍA"}

\author{
Viriam Leiva Díaz*
}

\begin{abstract}
RESUMEN
Este artículo presenta los resultados de una investigación de tipo cuantitativo, descriptivo y transversal. La población participante fue de 214 estudiantes que cursaban el segundo y cuarto año de la carrera de Licenciatura en Enfermería. Se aplicó un cuestionario autoadministrado, el cual fue previamente validado. El estudio evidenció que esta población estudiantil posee factores protectores para la prevención del consumo o el uso problemático de drogas; pero a la vez presenta factores de riesgo importantes como son: estilos de vida no saludables, consumo de drogas de personas cercanas a ellos como son familiar, amigos y compañeros.

Además se encontró que el perfil de consumo de drogas es similar al que se da en el ámbito nacional e internacional, destacándose el consumo de alcohol y tabaco, marihuana y cocaína.
\end{abstract}

PALABRAS CLAVES: COSTA RICA * DROGAS * CONSUMO * ESTUDIANTES * ENSEÑANZA SUPERIOR * ESCUELA DE ENFERMERÍA

Psicopedagogía y Escuela de Enfermería de la Universidad d Costa Rica.

viriaml@gmail.com

Este artículo forma parte del proyecto de investigación nro.200 aprobado por la Vicerrectoría de
Investigación, titulado "Factores sociales, culturales, personales, familiares y económicos asociados al fenómeno de las drogas en estudiantes de la Carrera de Licenciatura en Enfermería: un estudio desde la perspectiva de la Salud Internacional", Universidad de Costa Rica. 


\section{ABSTRACT}

This article presents the results of quantitative study, descriptive and transverse. The sample was of 214 students that studied their fourth year on the nursing degree. They answered a questionnaire which was previously validated.

The study evidenced that students possess certain factors that protect them against drug usage or the advance of drug habits; but at the same time they have risk factors which must be taken into consideration in prevention programs, such as: non-healthy life styles and drug abusage in people close to them, be it family, friends or classmates. Also we found that the used drug's profile, was similar to the one found nationally and internationally; in the first place of drug usage was alcohol, followed by tobacco, then by marihuana and cocaine.

KEYWORDS: COSTA RICA * DRUGS * CONSUMPTION * STUDENTS * HIGHER EDUCATION * ESCUELA DE ENFERMERÍA

\section{INTRODUCCIÓN}

Tanto en el nivel mundial como nacional, el uso y abuso de las drogas lícitas e ilícitas es una preocupación creciente en el ámbito político, social, económico y de la salud. No sólo por las modificaciones químicas o físicas que produce en el organismo producto de la acción directa en el sistema nervioso central alterando su estructura $y$ funcionamiento (World Health Organization, WHO, 2002); sino también en el ámbito familiar, social y mundial. Para esto basta con hacer mención del Informe de la Oficina contra la droga y el Crimen de la Organización de las Naciones Unidas (UNODC, por sus siglas en inglés) del 2004, que evidencia que, del total de la población mundial, 185 millones de personas son consumidoras de sustancias ilegales, cifra que se incrementa en un $5 \%$ para el año siguiente. Con respeto a las sustancias legales, la Organización Mundial de la Salud (OMS), para ese mismo año afirma que el alcohol es la tercera sustancia de mayor consumo de los países industrializados y el principal en los países en desarrollo; se estima que los consumidores ascienden a 2000 millones de personas en el mundo; seguido por el consumo de tabaco en aproximadamente 650 millones de la población mundial.

El consumo de drogas lícitas e ilícitas produce efectos físicos en un corto plazo (adicción), generando enfermedades y trastornos que llegan a ser degenerantes (incapacitantes) o mortales; todo esto en una población económicamente activa, lo cual repercute en el ámbito económico de un país, generando gastos por el pago de incapacidades temporales o permanentes; y en el ámbito social, ya que son daños en las relaciones interpersonales, pérdida del trabajo, desintegración familiar, entre otros.

En Costa Rica según datos del "Informe Consolidado sobre los costos del problema de las drogas ilegales en Costa Rica entre los años 2000-2003" (Pérez; Valencia; Rodríguez 2004), el primer lugar lo ocupó los gastos gubernamentales directos, es decir, en la inversión para la reducción de la oferta. A estos le siguieron los costos por pérdida de productividad y salud, que presentan un aumento leve pero sostenido hasta el 2002, y seguido por un descenso para el 2003 .

Según Pérez, Valencia y Rodríguez (2004) los datos anteriores podrían indicar por una parte que el problema de consumo interno se encuentra relativamente estable, lo cual justificaría la poca inversión estatal; o por otra, que el consumo sigue aumentando, pero que el Estado no ha considerado invertir más recursos en el tratamiento de los problemas generados por el consumo, pues, los delitos relacionados con narcotráfico exigen del Estado una inversión creciente de recursos, lo que explicaría el crecimiento de los costos totales.

En relación con lo anterior, el peso del aporte económico para el tratamiento de los 
problemas de adicción recae sobre las organizaciones no gubernamentales. Esto es claro al comparar la inversión gubernamental que oscila entre US\$ 40000 y uS\$ 100000 anuales (salvo en 2001 , que fue de us $\$ 1,72$ millones) y las inversiones no gubernamentales van de US\$1,7 millones a US\$ 3,1 millones; esto implica que el peso asumido por entidades no gubernamentales es alrededor 30 veces superior al asumido por el Estado en este rubro (Pérez, Valencia y Rodríguez, 2004).

Para facilitar una mayor comprensión de la problemática de drogadicción en Costa Rica se presenta los resultados de la última encuesta realizada por el Instituto sobre Alcoholismo y Farmacodependencia (IAFA), entre el año 2000 y el 2001, la cual es una réplica de la efectuada en los años 1990 y 1995, en una población entre los 17 y 70 años, por lo que se presentará la comparación de ambos estudios.

Se partirá del análisis del consumo de tabaco. Los datos mostraron que la prevalencia ${ }^{2}$ del uso para el 2001 fue de 17,2\%, lo cual muestra una reducción significativa del $1,1 \%$ del consumo activo de tabaco, comparados al estudio que le precedió en 1995. Se halló diferencias significativas con respecto al sexo, siendo los hombres $(41,1 \%)$ los que mostraron mayor consumo en relación a las mujeres (18,8\%). En lo que respecta a la edad de inicio de consumo de tabaco se ubicó al igual que el estudio anterior (1995) entre los 13 y 18 años. Además se encontró que, una vez iniciado el consumo de tabaco, es mayor la proporción de hombres $(56,2 \%)$ que continúan consumiéndolo que las mujeres $(43,6 \%)$.

Asociado a la prevalencia del consumo de benzodiacepinas de auto prescripción, es decir, sin que medie una receta médica, fue igual a la encontrada en el estudio anterior (2,2\%); se debe destacar que si bien el porcentaje de prevalencia es bajo, comparando toda la década, se muestra un aumento significativo, ya que para 1990 el consumo fue de 0,88\%. El mayor porcentaje de consumo estuvo ubicado en las personas de bajos recursos económicos y dentro

2 Cuando se habla de prevalencia se refiere al consumo de drogas en el último mes. de las principales razones expresadas para su uso estuvo en primer lugar como inductor del sueño (46\%) y en segundo lugar para disminuir la ansiedad (30\%).

En cuanto al consumo de estimulantes, no se encontró diferencias estadísticamente significativas entre el estudio anterior $(1,2 \%)$ $y$ el último realizado (1,9\%). La droga estimulante más usada es la tiamina con cafeína (70\%), que se caracteriza por ser de libre venta $y$ que genera algún grado de dependencia si media el abuso.

Otro grupo de drogas de importancia por su marcado efecto adictivo son los opiáceos, y dentro de ellos el más conocido es el sulfato de morfina, sobre el que se tiene un control estricto para su venta; sin embargo, hay otro grupo de medicamentos que no cuenta con dichos controles y se venden libremente, como son la codeína y el acetaminofén con codeína. Las personas que reportaron su uso $(85,3 \%)$ refirieron consumirlo única y exclusivamente para aliviar el dolor. Un dato importante es que la edad de inicio de uso de esta sustancia es relativamente menor en este estudio (17 años), que en el anterior (26,3 años).

Ahora bien, en lo que respecta al consumo de alcohol se encontró que para el 2000 la prevalencia de ingesta fue de un $54,3 \%$, la cual es menor que la mostrada en el estudio de 1995 (Bejarano, Ugalde, 2002). Se debe resaltar que esta baja puede estar asociada a un efecto positivo de los programas preventivos, sin embargo, esto no puede afirmarse categóricamente, dada la ausencia de evaluaciones a largo plazo de los mismos. En relación con la edad de iniciación del consumo de alcohol, se encontró que para el 2000 esta fue a los 15 años (38\%), siendo menor que en el estudio anterior, en que la edad se ubicó a los 18 años (60\%), con una reducción visible en la iniciación tardía del consumo (después de los 19 años). Por otro lado, los varones inician más tempranamente el consumo de alcohol que las mujeres, pero estas últimas ocupan el primer lugar en la iniciación tardía de la ingesta de alcohol.

En lo referente al consumo de drogas ilícitas, el primer lugar lo ocupa la marihuana (5,5\%), su consumo activo (en el último mes) se 
mantuvo estable ( $0,3 \%)$, lo que podría ser analizado como una mayor experimentación y estabilización de su consumo. La edad de iniciación fue significativamente menor (17,7 años) que el estudio anterior (18,5 años). No se encontró diferencias entre sexos en cuanto a edad de inicio. Además se encontró una asociación entre el consumo de marihuana, con el uso de tabaco y alcohol.

En cuanto a la cocaína, la prevalencia del consumo ha experimentado un aumento significativo. La edad de consumo para el presente estudio (19,9 años) fue estadísticamente significativa menor a la encontrada en el estudio de 1995 (22,1 años). El haber consumido cocaína alguna vez en la vida de una persona está fuertemente relacionado con ser consumidor activo de marihuana, alcohol, así como la condición de ser fumador.

Si entendemos que la formación en la prevención del uso y abuso de drogas es fundamental en los profesionales en salud, será comprensible también que estos profesionales hayan estado expuestos a la oferta de drogas, es por esto que se hace necesario investigar sobre los factores económicos, sociales, culturales y familiares que inciden en el consumo de drogas lícitas e ilícitas en estudiantes de segundo y cuarto año de la Carrera de Licenciatura en Enfermería de la Universidad de Costa Rica, constituyéndose esto en el objetivo de esta investigación y divulgación.

\section{MATERIALES Y MÉTODOS}

La investigación fue un estudio de tipo no experimental, cuantitativo, descriptivo, ya que pretende describir las variables en estudio, que se describirán más adelante. Esta investigación fue transversal, pues la información se recolectó durante el II semestre del 2007 (Hernández Fernández, Baptista, 2007).

Es importante destacar que en el presente artículo se ofrece un avance de los datos obtenido de la población estudiantil total de la Escuela de Enfermería de la Universidad de Costa Rica, que corresponden a 214 estudiantes que cursan II y IV año de la Escuela de Enfermería.
En relación con el establecimiento de las variables en estudio se utilizaron como base diferentes estudios que investigaban factores de riesgo y protectores en el uso y consumo problemático de las drogas (Carballo, García, Secades, Fernández, García, Erraste, 2004; Graña y Muñoz, 2000; Martínez, Fuertes, Ramos, Hernández, 2003; Organización Panamericana de la Salud, 2006, 2005; Cáseres, Salazar, Varela, Tobar, 2006). Entre las variables analizadas se encuentran las siguientes:

$\diamond$ Autoestima: es asociada como factor protector del consumo de drogas (Gutiérrez, E.; Aneiros, R. 1999); sin embargo, este atributo no cuenta con suficientemente evidencia científica, más bien Malcom (2004) y Rojas (2001), encontraron que una autoestima alta puede hacer que la persona se sienta confiada y aumente comportamientos de riesgo como consumir drogas.

$\diamond$ Aspectos psicológicos: la ansiedad, la depresión y el estrés, entre otros, están asociados al consumo de drogas, que se constituye en un medio rápido de alivio de las sensaciones corporales asociadas al malestar emocional, por sus efectos directos sobre el sistema nervioso central (Gutiérrez, Aneiros, 1999; Wu; Sterling; Weisner, 2004).

$\diamond$ Inhabilidad social e insatisfacción en las relaciones interpersonales: se refleja en la incapacidad de las personas para defender sus opiniones frente a otros, lo cual influye directamente en la dificultad que presenta un sujeto para rechazar la oferta de drogas; así como la atribución cultural que se le da al consumo de drogas como medio para ser aceptado por el grupo de iguales (Butters, 2004; Carballo; García; Secades; Fernández; García; Erraste, 2004).

$\diamond$ Habilidades emocionales: disminuyen la probabilidad de consumo, ya que, al desarrollar el autoconocimiento, la identificación, la expresión y el manejo de sentimientos, se logra mejorar el manejo del estrés y la ansiedad de una manera positiva (Griffin; Scheier; Botvin; Díaz, 2001).

$\diamond$ Los preconceptos y la valoración de letalidad de las drogas: la baja percepción de riesgo del uso de drogas, así como de sus efectos y 
consecuencias, lleva al consumo y abuso de las mismas (Pons, J. y Borjano, E. 1999).

$\diamond$ La espiritualidad: entendida como la creencia en una moralidad y ética, es un factor protector que previene el consumo inicial y facilita la abstinencia en los procesos de recuperación de la adicción (Navarro, J., 2000).

$\diamond$ El maltrato en todas sus formas y la disfunción familiar: estos han sido reconocidos como un augurio de consumo de drogas. El primero se ha asociado a la inducción temprana y el otro a los primeros consumos en adolescentes (Pons; Borjano, 1999).

$\diamond$ La relación con la persona consumidora: el sujeto que consume ejerce presión social sobre los no consumidores, ya que son en muchos casos los significantes sociales principalmente en los jóvenes. Estos consumidores funcionan como modelos a seguir dentro del aprendizaje social $y$ se forman en una trampa conductual para el inicio del consumo de drogas de los demás.

Para la recolección de la información de las variables previamente descritas se construyó y validó un cuestionario con preguntas cerradas, codificadas. Este se dividió en seis partes: información general, sociodemográfica, económica, personal, familiar y sociocultural y consumo de drogas.

Antes de aplicar el cuestionario, los sujetos fueron informados sobre su participación en el estudio, los fines del mismo, así como la confidencialidad de sus respuestas, todo esto se concretó en la firma del Consentimiento Informado. La recolección de información se llevó a cabo durante dos meses de manera grupal y auto-administrada.

Para el análisis de resultados se utilizó el Statistical Package for Social Sciences [SPSS] versión 13.0 para Windows. Se realizaron tablas de distribución de frecuencias simples para las variables en estudio.

\section{RESULTADOS DE LA INVESTIGACIÓN}

Para iniciar la presentación de este apartado se debe hacer mención que, si bien es cierto la población meta estuvo constituida por 214 estudiantes, el paquete estadístico utilizado dio 30 estudiantes como perdidos ya que no respondieron a un importante número de preguntas del cuestionario, por lo que se presentarán los resultados de los 184 estudiantes restantes.

La población de la Carrera de Enfermería es eminentemente femenina, como lo evidencian los datos, en que de las 184 encuestas, 160 son del sexo femenino. Además, el $75,1 \%$ de la población estudiantil son menores de 24 años de edad, destacándose que el 65,5\% tienen 20 o menos años de edad, es decir, lo que la OMS clasifica como adolescencia media (Krauskopf, 2002). Asimismo, en lo correspondiente al estado civil un $80,5 \%$ son solteros.

Una variable que es considerada como un elemento protector para la prevención del consumo de drogas es el aspecto familiar, distinguiéndose la estabilidad de la misma como un elemento positivo. Respecto a esto, en el cuadro 1 se evidencia que en un importante número de los encuestados se mantiene el perfil de familia nuclear, destacándose la convivencia en tiempo no lectivo, donde los mismos viven con sus padres $y$ hermanos.

\section{CUADRO 1}

DISTRIBUCIÓN DE LA POBLACIÓN ESTUDIANTIL SEGÚN PERSONA CON QUIEN VIVE EN TIEMPO LECTIVO Y NO LECTIVO, 2007

\begin{tabular}{lcc}
\hline \multicolumn{1}{c}{ PERSONA } & $\begin{array}{r}\text { EN TIEMPO } \\
\text { LECTIV }\end{array}$ & $\begin{array}{r}\text { EN TIEMPO NO } \\
\text { LECTIVO }\end{array}$ \\
\hline Solo & $3 \%$ & $1,6 \%$ \\
Papá & $25,5 \%$ & $50,1 \%$ \\
Mamá & $37,7 \%$ & $67,3 \%$ \\
Hermanos & $38,8 \%$ & $63,9 \%$ \\
Hijos & $2,8 \%$ & $2,7 \%$ \\
Pareja & $5,7 \%$ & $4,5 \%$ \\
Familiares & $11,8 \%$ & $11,3 \%$ \\
Compañeros & $22,3 \%$ & $0,8 \%$ \\
Amigos & $18,1 \%$ & $1,2 \%$ \\
\hline
\end{tabular}

Siempre en lo referente a la variable familiar, se destaca la escolaridad de los padres de los encuestados, un $29,4 \%$ poseen estudios universitarios, un $16,5 \%$ tienen secundaria 
completa y un $15,9 \%$ primaria completa. El nivel de escolaridad de los padres, está directamente relacionado con la posibilidad de ingreso económico de una familia, así como las oportunidades de acceso a la educación superior y prevención de la deserción escolar en los jóvenes (EDNA, 2005).

La fuente de ingreso económico familiar del grupo encuestado proviene principalmente del salario (65,9\%), y la persona que aporta dicho ingreso es la madre en primer lugar con un $56,2 \%$, seguido del padre con un $43,5 \%$. Cabe mencionar que ambas opciones no fueron excluyentes, es decir, que puede ser el padre, la madre o ambos. El resto del porcentaje se distribuye entre hermanos y pareja. El ingreso mensual familiar, se encuentra en un $42 \%$ entre 100 mil a 300 mil colones (esto es entre $\$ 200$ y $\$ 600)$, el resto está distribuido entre menos de 100 mil colones $(16,2 \%)$, más de 300 mil colones $(21,3 \%)$ y más de 600 mil colones $(4,2 \%)$.

Es importante notar que un factor que influye en el aspecto económico familiar es que los cursos que se brindan en la Carrera de Licenciatura en Enfermería son de horario vespertino, por lo que los estudiantes tienen dificultad para combinar los estudios con actividades laborales, que podría de esta manera compensar los gastos económicos en que tienen que incurrir para el pago y manutención de sus estudios. Por tanto, no es de extrañar que, de los 184 encuestados, un $75,8 \%$ de la población estudiantil no trabajan; un 10,1\% laboran $y$ de estos un $6,2 \%$ realizan actividades no registradas, es decir, que no poseen las garantías otorgadas por ley como son: pago de salarios mínimos, pago de seguro social, el cual cubre enfermedad, maternidad, invalidez $y$ muerte. De los jóvenes que trabajan un 35,6\% lo hace en tiempo parcial, es decir menos de 6 horas diarias.

Por otra parte, la ubicación geográfica de la Escuela de Enfermería y la concentración de las experiencias clínicas necesarias para el aprendizaje en el área metropolitana, obligan al estudiantado a trasladarse de sus lugares de origen $y$ alquilar una habitación o compartir otros inmuebles con compañeros cerca de la Sede Central de la Universidad de Costa Rica. En el cuadro 2 se muestra que en la provincia de San José un $58,8 \%$ de los estudiantes reside en ella $y$ tan solo un $30,8 \%$ procede de esta provincia, ya que la Sede Central se encuentra ubicada en dicha provincia.

CUADRO 2

DISTRIBUCIÓN DE LA POBLACIÓN ENCUESTADA SEGÚN PROVINCIA DE PROCEDENCIA Y DE RESIDENCIA, 2007

\begin{tabular}{ccccc}
\hline \multirow{2}{*}{ PROVINCIA } & \multicolumn{2}{c}{ PROCEDENCIA } & \multicolumn{2}{c}{ RESIDENCIA } \\
\hline & NÚMERO & PORCENTAJE & NÚMERO & PORCENTAJE \\
\hline San José & 91 & $40,6 \%$ & 114 & $49 \%$ \\
Alajuela & 31 & $13,3 \%$ & 24 & $10,9 \%$ \\
Cartago & 21 & $9,2 \%$ & 23 & $10,1 \%$ \\
Heredia & 21 & $9,3 \%$ & 20 & $8,7 \%$ \\
Puntarenas & 16 & $6,7 \%$ & 7 & $3,2 \%$ \\
Guanacaste & 12 & $5 \%$ & 4 & $3,7 \%$ \\
Limón & 4 & $1,7 \%$ & 3 & $1,3 \%$ \\
\hline
\end{tabular}


Todo lo anterior lleva a esta población a la solicitud de beca, a la que puede aspirar cualquier estudiante de bajos recursos económicos dentro de la Universidad de Costa Rica.

Así tenemos que, del total de encuestados, un $78,2 \%$ disfrutan de algún tipo de beca, de estos un $49,3 \%$ poseen beca 11 , esta incluye la exoneración del pago de matrícula, préstamo de libros semestral o anualmente, pago de la alimentación y en algunos casos, pago de una mensualidad.

Un $28,3 \%$ tienen beca 10 . En esta categoría de beca, el alumnado recibe igual beneficio que la beca 11 con excepción de pago de una mensualidad. Adicionalmente, un $10,6 \%$ recibe algún tipo de beca desde la 9 a la 1 , estas becas escalonan porcentualmente el pago de la matrícula, por último, cabe mencionar que tan solo $11,8 \%$ no tienen beca. De los que reciben algún tipo de beca un $48,8 \%$ la consideran insuficiente para cubrir los gastos que conlleva el estudio, así como su manutención.

Ahora bien, se exploró en el área de intereses $y$ expectativas personales, aspectos de la Carrera de Enfermería. Se encontró una motivación intensiva asociada a la finalización de estudios y mejora en la expectativa económica futura, esto se asocia con su grado de satisfacción, un 55,9\% refieren estar satisfechos con su elección profesional y en regular satisfacción un $23 \%$.
Cabe mencionar que un 39,6\% pensó en algún momento renunciar a los estudios; sin embargo, conforme aumenta el avance de la carrera, disminuye el deseo de abandono de la misma. Así, del 39,6\% que manifestó en algún momento deseo de deserción, disminuyó a un $25,1 \%$ en el primer año y en el segundo año a un $19,2 \%$. Esta tendencia descendiente puede estar asociada a un conocimiento mayor de la profesión, esto por cuanto la carrera de enfermería se ha caracterizado porque el estudiantado ingresa con la expectativa de traslado a otras carreras afines al área de la salud; sin embargo, a partir del II ciclo del primer año deben tomar cursos propios de la carrera lo cual coincide con la disminución del deseo de abandono de la misma y mayor satisfacción.

Esta población estudiantil opina que su desempeño en la carrera es muy bueno o bueno $(59,7 \%)$ y un $19,3 \%$ lo considera regular o deficiente. Estos datos son ratificados, pues en el último semestre previo a la encuesta un $60,4 \%$ ganaron todas las materias, un 10,6\% presentó examen de ampliación y ganó todas las materias matriculadas; $y$ un $29 \%$ presentó examen de ampliación pero no ganaron todas las materias.

Ahora bien, otra variable importante de analizar fue los estilos de vida saludable del estudiantado, destacándose dentro de estos el uso del tiempo no lectivo. En el cuadro 3 se presenta los sitios más frecuentados por estos.

CUADRO 3

DISTRIBUCIÓN PORCENTUAL DE LUGARES VISITADOS POR LOS ESTUDIANTES EN TIEMPO NO LECTIVO, SEGÚN FRECUENCIA, 2007

\begin{tabular}{cccccc}
\hline LUGAR & SIEMPRE & MUCHA FRECUENCIA & FRECUENTEMENTE & $\begin{array}{c}\text { POCO } \\
\text { FRECUENTE }\end{array}$ & $\begin{array}{c}\text { NO } \\
\text { FRECUENCIA }\end{array}$ \\
\hline Biblioteca & 8,9 & 22,5 & 23,6 & 19,9 & 10,1 \\
Soda & 9,6 & 17,6 & 23,4 & 27,3 & 8,0 \\
Centro cómputo & 9,6 & 26,2 & 21,0 & 17,0 & 11,6 \\
Asociación de & 1,3 & 3,1 & 4,0 & 18,7 & 58,3 \\
estudiantes & 9,7 & 12,5 & 19,7 & 25,5 & 17,9 \\
Zona verde & 9,3 & 16,2 & 11,6 & 45,2 \\
Sala CATE* & 2,2 & 2,2 & 5,7 & 16,7 & 58,6 \\
Deporte & 1,7 & & & & \\
\hline
\end{tabular}

\footnotetext{
* Centro Audiovisual y Tecnología en Enfermería.
} 
En tiempo no lectivo dentro del campus, el lugar más frecuentado por la población de cuarto año es la sala del CATE (30,8\%), este es un centro de simulaciones, donde estos educandos pueden realizar procedimientos de enfermería; en segundo lugar se mencionan los centros de cómputo (29,0\%), la biblioteca $(22,4 \%)$ y la soda $(16,8 \%)$ y dentro de los menos visitados están las zonas verdes (9,3\%), la Asociación de Estudiantes (0,9\%) y las áreas deportivas $(0,9 \%)$.

De igual manera, dentro de los lugares más visitados en tiempo no lectivo por el estudiantado de segundo año, se ubica en primer lugar la biblioteca $(67,3 \%)$, seguido por la soda (63\%) y por último los centros de cómputo $(61,5 \%)$ y entre los menos visitados se encuentra el CATE (94,9\%), la Asociación de Estudiantes $(92,4 \%)$ y las áreas deportivas $(89,1 \%)$.

En lo que respecta a las redes de apoyo con que cuentan la población estudiantil seleccionada, un $74,2 \%$ del estudiantado refieren recibir apoyo emocional en cantidad y calidad suficiente. El cuadro 4 muestra a las personas que buscan en caso de tener un problema.

\section{CUADRO 4}

DISTRIBUCIÓN DE LA POBLACIÓN ESTUDIANTIL SEGÚN PERSONA A LA QUE ACUDEN EN CASO DE UN PROBLEMA, 2007

\begin{tabular}{ccc}
\hline PERSONA & NÚMERO & PORCENTAJE \\
\hline Ninguna & 5 & 2,7 \\
Mamá & 145 & 78,8 \\
Papá & 59 & 32,0 \\
Hermanos & 67 & 36,4 \\
Pareja & 85 & 46,2 \\
Amigos & 130 & 70,6 \\
Familiares & 34 & 18,5 \\
Profesor & 19 & 19,3 \\
Otros & 5 & 2,7 \\
\hline
\end{tabular}

Este cuadro evidencia que en primera instancia acuden a la madre dentro de su grupo familiar $y$ fuera de este, a los amigos y la pareja. Esto es especialmente importante ya que la consejería que brinda el grupo de iguales puede tener impacto positivo o negativo en las acciones de quien solicita apoyo.

Ahora bien, una variable que refleja la auto-percepción y la autoestima que posee una persona dentro de un grupo determinado es la relación interpersonal. La juventud tiene claramente definida esta área, es así como un 80,1\% perciben que su opinión y sentimientos dentro del grupo de iguales o de amigos son aceptados $y$ un $59,8 \%$ manifiestan que pueden expresarlo aún cuando el grupo acepte o no dicha opinión.

A pesar de las fortalezas antes encontradas, un grupo de estudiantes tienen factores de riesgo interpersonal para el consumo de drogas; así los datos reflejan que a un $10,4 \%$ de esta población le es difícil hacer amigos, un 11,2\% se sienten relegados por el grupo de amigos o de pares y un $13,4 \%$ se consideran fácilmente influenciables.

Seguidamente, otra variable digna de destacar como un factor protector en el consumo de drogas es la religión. Un 60,8\% del grupo de encuestados profesan la religión católica, esto es coincidente con el perfil religioso de los $y$ las costarricenses. Un 16,9\% practican otras religiones (evangélica, testigo de Jehová, protestante $y$ otras) y un $10,6 \%$ refieren no ejercer ninguna religión.

Todos los aspectos anteriormente mencionados son importantes para la comprensión de una población frente al consumo de drogas. A continuación se brinda los datos obtenidos sobre la temática del consumo de drogas.

De las personas seleccionadas, los que manifestaron mayor porcentaje de consumo de drogas lícita e ilícita alguna vez en su vida, fue el grupo de segundo año (62,2\%), seguido por el estudiantado que cursan el cuarto (43,0\%); cabe destacar que en este grupo hubo un mayor índice de no respuesta.

De la población encuestada que manifestaron no haber probado drogas, describieron como causas para no consumir: el conocer los efectos secundarios de las drogas (24,7\%), que no les llamaba la atención (26,6\%), y por último, el no necesitar consumirla para desempeñarse socialmente $(23,1 \%)$.

Ahora bien, tanto aquellos estudiantes que nunca han consumido drogas, así como los 
que han abandonado su uso y los que tienen un consumo activo, coincidieron que los amigos $y$ compañeros (cuadro 5) fueron los que le ofrecieron drogas por primera vez, ambos hacen referencia al grupo de iguales (grupo etario).
Asociado a esto, un 41,3\% del estudiantado manifestó que se les ofreció drogas por primera vez alrededor de los 15 años de edad, algunos inclusive refirieron que se les ofreció antes de esta edad.

CUADRO 5

DISTRIBUCIÓN DE LA POBLACIÓN GENERAL, SEGÚN RELACIÓN DE PARENTESCO DE LA PERSONA QUE OFRECIÓ DROGAS POR PRIMERA VEZ

\begin{tabular}{cccc}
\hline RELACIÓN PARENTESCO & $\begin{array}{c}\text { PORCENTAJE EN TODA LA } \\
\text { POBLACIÓN }\end{array}$ & $\begin{array}{c}\text { PORCENTAJE EN } \\
\text { LA POBLACIÓN QUE } \\
\text { CONSUMIÓ }\end{array}$ & $\begin{array}{c}\text { PORCENTAJE EN } \\
\text { LA POBLACIÓN QUE } \\
\text { CONSUME }\end{array}$ \\
\hline Amigos & 19,0 & 49,9 & 40,4 \\
Compañeros & 10,9 & 13,1 & 28,7 \\
Familiares & 4,2 & 18,5 & 5,1 \\
Pareja & 2,1 & 3,9 & 2,6 \\
Otros & 1,9 & 0,9 & 0,9 \\
\hline
\end{tabular}

Con respecto a los indicadores sobre uso problemático de bebidas alcohólicas en la población que consume o consumió alcohol se encontró que mayoritariamente $(75,5 \%)$ no refirieron problemas en cuanto al consumo de alcohol, pues en el rubro en que se presentó mayor porcentaje de respuesta afirmativa $(27,2 \%)$ fue en el que exteriorizaron sentir que deberían disminuir la cantidad de bebidas 0 parar de beber. Es importante destacar que hay siete estudiantes que exhiben un uso problemático de alcohol.

Asimismo, la población encuestada se encuentra mayoritariamente en desacuerdo $(71,1 \%)$ con el uso de drogas ilícitas en actividades religiosas, familiares, o en reuniones informales y formarles. Este porcentaje en desacuerdo disminuye porcentualmente en poco más de dos tercios frente al uso de tabaco $(37,1 \%)$, un bajo porcentaje de estudiantes se mostró en desacuerdo con respecto al consumo de alcohol (18,3\%) en dichas actividades sociales. Esto puede estar asociado a que en las actividades familiares, religiosas o sociales que los estudiantes frecuentan se ofrece alcohol y tabaco.

En contraste con el anterior epígrafe, el consumo de drogas lícitas es frecuente en actividades sociales informales $y$ formales; así el mayor consumo de bebidas alcohólicas se da en las actividades familiares $(47,4 \%)$ con respecto a las religiosas (41\%), el uso del tabaco tiene una relación inversa al alcohol es decir, es mayor en las actividades religiosas $(59,6 \%)$ que en las actividades familiares (47,8\%). Es importante destacar que algunas de las personas encuestadas refirieron que frecuentemente se ofrecen drogas ilícitas en las actividades en que participan (5,5\%).

En el cuadro $6^{3}$ se muestra las razones por las cuales se consume drogas en el ámbito social, entre las más frecuentes se encuentran en primer lugar el consumo de drogas por diversión $(63,9 \%)$ o placer $(43,9 \%)$, en segundo lugar para acompañar a la pareja(42\%) o amigos $(37,4 \%)$ y en tercer lugar para relacionarse con otras personas $(16,8 \%)$. Todas estas razones están asociadas a la esfera social en donde las relaciones interpersonales tienen un papel preponderante, incluso según el saber popular de que el uso de drogas ayuda a desinhibirse socialmente, genera respeto $y$ aprecio dentro del grupo social en que se desarrolla la persona.

3 Los datos presentados en el cuadro 6 no son mutuamente excluyentes, se podía indicar más de una opción. 
CUADRO 6

DISTRIBUCIÓN DE MOTIVO DE CONSUMO DE DROGAS A NIVEL SOCIAL, SEGÚN PORCENTAJE, 2007

\begin{tabular}{lcc}
\hline & IV AÑO & II AÑO \\
\hline MOTIVO & PORCENTAJE & PORCENTAJE \\
\hline Pertenecer al grupo & 15,9 & 22,7 \\
Relacionarse con otros & 16,8 & 31,9 \\
Diversión o placer & 43,9 & 63,9 \\
Aumentar el desempeño & 4,7 & 5,9 \\
sexual & & \\
Ser respetado & 7,5 & 12,6 \\
Ser más apreciado & 7,5 & 11,8 \\
Acompañar a la pareja & 37,4 & 42,0 \\
o amigos & & \\
\hline
\end{tabular}

Un dato importante que refleja la problemática de la drogadicción y su efecto en la esfera social, es la relación que se da entre el consumo de drogas y los accidentes de tránsito. En el grupo encuestado esta relación fue muy baja, tan solo un 2,5\% refirieron haber estado implicados en un accidente de tránsito, asociado al uso de alguna sustancia lícita o ilícita.

Aún cuando los datos anteriores son halagüeños, se debe destacar que la población estudiantil estudiada expresa conductas de riesgo que los expone a situaciones poco saludables en su estilo de vida. Dentro de estas conductas se encuentran: ingesta de alimentos con alto contenido en grasa $(26,8 \%)$, consumo de alcohol $(23,5 \%)$, sedentarismo $(20,4 \%)$, relaciones coitales sin protección $(12,3 \%)$, consumo de cigarrillo convencionales $(14,0 \%)$ y finalmente inhalación de solventes (13,5\%).

En otro asunto, es conocido que el consumo de drogas en la familia es un factor que puede predisponer al uso y abuso de drogas en la juventud, por lo que se indagó al respecto. La droga lícita más utilizada con una frecuencia de no menos de tres veces por semana $y$ sin fines médicos, es el tabaco, seguido por el alcohol. Entre las drogas ilícitas, los datos señalaron porcentajes muy bajos de marihuana, inhalantes, tranquilizantes, crack y cocaína.

Asimismo, los familiares del grupo encuestado que presentan un consumo mayor de bebidas alcohólicas son en primer lugar los tíos y tías (18,7\%), seguidos por el padre y los hermanos o hermanas $(13,1 \%)$.

En lo que respecta al consumo de tabaco, para el grupo de encuestados de II año, las madres (25\%) ocupan el primer lugar como consumidoras, seguidas por los padres $(6,5 \%)$. Para el estudiantado de IV año, el primer lugar lo ocupan los hermanos, hermanas, los tíos $y$ tías (14,0\%). Cabe rescatar dos aspectos importantes: primero, en cuanto a las drogas lícitas (alcohol y tabaco) los tíos, así como las tías, ocupan el primer lugar; y segundo, se encuentra predominio del consumo de tabaco en las madres de los jóvenes de segundo año. Ambos resultados denotan cambios culturales en cuanto al consumo de tabaco y alcohol por parte de las mujeres, esta práctica fue predominantemente de uso de los varones.

Como se ha podido observar en la encuesta un 58,2\% refieren tener al menos un familiar que consume drogas. De estos un $38,3 \%$ mencionan que las causas para que el familiar iniciara el consumo de drogas fueron los problemas familiares, con amigos o en el trabajo. Un 13,7\% refirió que dicho familiar se ha visto envuelto en un accidente de tránsito en que medió el consumo de una droga.

Desglosando el efecto que produce el consumo problemático de drogas entre los parientes cercanos, se tiene los problemas familiares (44,3\%), la pareja (25,4\%), los hijos (20\%), los laborales $(16,6 \%)$ y por último los estudios $(9,8 \%)$. Todos estos datos apuntan a las situaciones que se viven alrededor del consumo de drogas que afecta no solo al individuo si no al grupo familiar nuclear y extenso. Lo más preocupante es que tan solo un $10,2 \%$ de los familiares del grupo encuestado han buscado algún tipo de ayuda.

Como se describió en los párrafos previos, dentro de las personas que le ofrecieron algún tipo de droga a las personas encuestadas se encuentran los amigos o compañeros, por lo que es importante conocer la situación de consumo de drogas dentro de estos. En los resultados se evidenció que dentro de los amigos más cercanos, el consumo de bebidas alcohólicas ocupa el primer lugar $(88,2 \%)$, seguido por el 
tabaco $(84,9 \%)$, en tercer lugar la marihuana $(28,6 \%)$ y en el cuarto lugar se encuentran la acetona, heroína y solventes (17,6\%).

Con base en toda esta información a continuación se ofrece el apartado de discusión de los datos.

\section{DISCUSIÓN}

En los datos presentados en el apartado anterior, se evidenció un perfil similar al nacional en cuanto al uso de drogas, con una edad de iniciación asociada a la pubertad y un consumo problemático que acarrea situaciones críticas en el ámbito personal, familiar, social y laboral (Bejarano, 2003).

Además, se encontró que la droga más consumida por estudiantes universitarios es el alcohol, seguida por el tabaco, así como un bajo consumo de drogas ilícitas, dentro de estas las más consumidas son la marihuana y la cocaína, el mismo perfil descrito tanto en la esfera nacional como internacional (Bejarano, 2003; OMS, 2005).

El ofrecimiento de drogas, así como el inicio del consumo de las mismas, se da en edades menores a los 18 años. Esto coincide con los hallazgos encontrados por Bejarano (2003) y Camacho (2005).

Según los factores de riesgo y protección establecida por Arkowitz, H.; Hinton, R.; Perl, J. y Himadi, W.(1978), el grupo de estudiantes encuestados poseen más factores protectores que de riesgo, dentro de estos podemos mencionar la estabilidad de la estructura y dinámica familiar, que satisface los procesos y funciones de la familia, autoestima y sentido de pertenencia familiar social, así como la posibilidad de proyectos de vida viables en congruencia con la escala de valores morales apropiados.

Dentro de los factores de riesgo que presenta la población se encuentran consumo de alcohol $y$ drogas, riesgo de enfermedades de transmisión sexual asociadas a prácticas riesgosas, inadecuado empleo del tiempo libre, dificultad de acceso económico que repercute en la estabilidad de la educación y consecución de metas.

Sobre el comportamiento de consumo de drogas lícitas e ilícitas, que incluye tanto el alcohol como el tabaco, fue consecuente con los hallazgos encontrados por Camacho, I. (2005); Cáseres, D.; Salazar, I.; Varela, M.; Tobar, J.(2006); Zárate, M.; Zavaleta, A.; Danjoy, D.; Chanamé, E.; Prochazka, R.; Salas, M.; Maldonado, V.(2006) en términos de que el consumo mayor por parte de las personas encuestadas, sus amigos y familiares, se encuentra predominantemente asociado al uso del alcohol y el tabaco como droga lícita, y la marihuana y cocaína como drogas ilícitas.

La población encuestada que consumen algún tipo de droga, refieren no tener problemas derivados del consumo, dicho comportamiento se extiende al grupo de amigos, no así dentro de los familiares los cuales sí presentan un consumo problemático de drogas, que ha derivado en problemas familiares, laborales y personales.

Los resultados revelan además que el consumo de drogas tanto lícitas como ilícitas tiene un importante componente social, las expectativas positivas dadas al consumo primordialmente del alcohol como un desinhibidor social y su capacidad para ayudar a enfrentar situaciones difíciles, tanto en el entorno social como familiar, $y$ a la mejora de las relaciones vinculares interpersonales, podría convertirse en un factor causal importante del mantenimiento de la conducta de beber o del desarrollo abusivo del alcohol (Mora-Ríos, J.; Natera, G.; Villatoro, J.; Villalvazo, R., 2000) en los jóvenes que dicen consumirlo.

La presencia de situaciones perturbadoras como dificultades en la familia, el trabajo o la pareja, parece estar asociado a la iniciación o intensificación del consumo de drogas en los familiares $y$ amigos del estudiantado encuestado, reforzado este consumo con los preconceptos y sobre valoraciones positivas que genera su uso.

Aún cuando no se puede en este momento establecer con valor predictivo la relación entre tener personas cercanas consumidoras o no, este aspecto sí ha sido estudiado. En esta investigación, se considera relevante rescatar que esta población posee familiares que presentan problemas en el uso de drogas, así como un porcentaje importante de amigos que también ingieren frecuentemente algún tipo de droga. 
Como lo manifiesta Cáseres; Salazar; Varela; Tobar (2006) uno de los mejores factores que predicen la utilización de drogas es el hecho de tener amigos o familiares que ingieran y por el contrario, se convierte en un factor protector, en aquellos jóvenes que tienen desaprobación por parte de su grupo de iguales en lo referente al uso de mismas.

\section{CONCLUSIONES}

$\diamond$ La edad de inicio (16 años) de consumo de drogas en la población encuestada fue similar al promedio nacional (17,13 años) (Bejarano, 2003).

$\diamond$ Las personas que ofrecieron o brindaron la primera droga a los estudiantes fueron amigos o compañeros.

$\diamond$ Las causas que refieren los jóvenes para el consumo de drogas son la diversión o placer, para acompañar a la pareja o amigos $y$ en tercer lugar el relacionarse con otras personas.

$\diamond$ Unido a lo anterior, el consumo de drogas por amigos y familiares puede ser un factor de riesgo en los estudiantes encuestados para el consumo de drogas y mantenimiento de conductas riesgosas. Esto sumado a una percepción de consecuencias del consumo con expectativas positivas, como reducción de ansiedad, relajación, facilitador de las interacciones sociales, entre otras.

$\diamond$ Se evidenció prácticas riesgosas para un estilo de vida saludable como es el sedentarismo, el consumo de alimentos ricos en grasas, la poca o ninguna actividad física con periodicidad, relaciones coitales sin protección, etc.

$\diamond$ Entre los familiares que consumen algún tipo de droga sin fines médicos hay diferencias significativas, destacándose el consumo frecuente entre los hermanos, tíos y padres.

$\diamond$ Evidencian entre el estudiantado, una tolerancia y aceptación mayor del consumo de alcohol y tabaco en actividades familiares, sociales y religiosas, que el consumo de drogas ilícitas. Esto está asociado a la concepción del término licito (legal) o ilícito (ilegal). $\diamond$ El estudiantado muestra conductas protectoras entre las que se destacan: estabilidad familiar, proyectos de vida, relaciones grupales estables, etc.

$\diamond$ Los jóvenes que ingieren alcohol refieren no tener un consumo problemático del mismo.

\section{BIBLIOGRAFÍA}

Arkowitz, H.; Hinton, R.; Perl, J. y Himadi, W. "Treatment strategies for dating anxiety in college men based on real-life practice". The Counseling Psychologist 7. 1978: 41-46.

Bejarano, J.; Ugalde, F. (2002). Consumo de drogas en Costa Rica. Resultados de la Encuesta Nacional del 2000-2001. Costa Rica: Instituto sobre Alcoholismo y Farmacondependencia (IAFA), 2003.

Butters, J. E. "The impact of peers and social disapproval on high-risk cannabis use: Gender differences and implications for drug education". Drugs: Education, Prevention and Policy 11. 2004: 381-390.

Cáseres, D.; Salazar, I.; Varela, M.; Tobar, J. "Consumo de drogas en jóvenes universitarios y su relación de riesgo y protección con los factores psicosociales". Univ.Psychol 5 (3), octubre-diciembre. Calí: Universidad Javeriana, 2006.

Camacho, I. "Consumo de alcohol en universitarios: relación funcional con los factores sociodemigráficos, las expectativas y la ansiedad social". Acta colombiana de psicología 8 (1), marzo. Bogotá: Universidad Católica Colombiana, 2005.

Carballo, J. L. et ál. "Construcción y validación de un cuestionario de factores de riesgo interpersonales para el consumo de drogas en la adolescencia”. Psicothema 16, 2004: 674-679. 
Chavarría, M.; Jiménez, M.; Villalobos, S. "Farmacodenpendencia en el medio universitario". [Tesis de Licenciatura en Trabajo Social]. Escuela de Trabajo Social. Universidad de Costa Rica, 1993.

EDNA. Quinto Estado de la niñez y la adolescencia. Costa Rica, 2005.

Gutierrez, E.; Aneiros, R. Toxicomanías $y$ adolescencia: Realidades $y$ consecuencias. La Habana: Clínica del adolescente, 1999.

Graña, J.; Muñoz, M. "Factores de riesgo relacionados con la influencia del grupo de iguales para el consumo de drogas en adolescentes". Psicología conductual 8. 2000: 19-32.

Griffin, K.; Scheier, L.; Botvin, G. y Díaz, T. "Protective role of personal competence skills in adolescent substance use: Psychological well-being as a mediating factor". Addictive Behaviors 15. 2001: 194-203.

Hernández, R.; Fernández, C.; Baptista, P. (2006). Metodología de la investigación. Cuarta edición. México: Editorial McGraw-Hill Interamericana, 2007.

Krauskopf, Dina. Participación social y desarrollo en la adolescencia. Segunda edición.Costa Rica: Editorial EUNED, 2002.

Malcolm, B. "Evaluating the effects of selfesteem on substance abuse among homeless men". Journal of Alcohol y Drug Education 48. 2004:39-61.

Martínez, J.; Fuertes, A.; Ramos, M. y Hernández, A. "Consumo de drogas en la adolescencia: importancia del afecto $y$ la supervisión parental". Psicothema 15. 2003: 161-166.
Mora-Ríos, J.; Natera, G.; Villatoro,J.; Villalvazo, R. "Validez factorial del cuestionario de expectativas hacia el alcohol (AEQ) en estudiantes universitarios". Psicología Conductual 8. 2000: 319-328.

Navarro Botella, J. Factores de riesgo $y$ protección de carácter social relacionados con el consumo de drogas. Madrid: Plan Municipal contra las Drogas. Ayuntamiento de Madrid. 2000. [En línea].En: <http://www.mir.es/pnd/ publica/html/otras.htm>

Oficina contra la droga y el crimen de la Organización de las Naciones UnidasUNODC. World Drug Report 2005. En:<http://www.unodc.org/unodc/en/ world_drug_report.html> [Recuperado el 20 de abril del 2008].

Oficina contra la droga y el crimen de la Organización de las Naciones Unidas (2006). UNOdC Colombia. Programa antidrogas. En: <http://www.unodc.org/ colombia/es/ drug_programme.html> [Recuperado 5 de marzo de 2008].

Pérez, A.; Valencia, J.; Rodríguez, C.Informe Consolidado sobre los costos del problema de las drogas ilegales en Costa Rica: 2000-2003.Washington: CICAD, 2004. En: <http://www.cicad.oas.org/oid/ MainPage/Costs/Informe\%20Final\%20 Costa\%20Rica.pdf> [Recuperado el 12 de abril de 2008].

Pons, J. y Borjano, E. El consumo abusivo de alcohol en la adolescencia: un modelo explicativo desde la psicología social. Valencia: Plan Nacional sobre Drogas/ Universidad de Valencia. 1999 [En línea]. En:<http://www.mir.es/ pnd/publica/ html/otras.htm >

Rojas, M. "Factores de riesgo y de protección que han sido identificados en adolescentes consumidores de SPA". En: A. Zabaleta. Factores de riesgo y 
protección en el consumo de drogas en la juventud. Perú: Centro de Información y Educación para la Prevención del Abuso de Drogas, 2001.

World Health Organization. The World Health Report. 2002. En:<http://www.who.int/ whr/2002/media_centre/en/index.html> [Recuperado el 25 de noviembre de 2007].

World Health Organization. The world health report: Shaping the future. Switzerland: World Health Organization, 2003.

World Health Organization. Global status report on alcohol 2004. En: <http:// www.who.int/ substance_abuse/ publications/alcohol/en/ index.html> [Recuperado el 13 de noviembre de 2007].

Wu, L.; Sterling, S.; Weisner, C. "Family environment factors and substance abuse severity in an HMO adolescent population". Clinical Pediatrics 43. 2004: 323-333.

Zárate, M. et ál. "Prácticas de consumo de tabaco y otras drogas en estudiantes de ciencias de la salud de una universidad privada de Lima, Perú". Invest. Educ. enferm. 24 (2), julio-diciembre. 2006: 72-81. 